\title{
Errors in the Area Determination of Incompletely Resolved Chromatographic Peaks
}

\author{
V.R. Meyer \\ University of Bern, Institute of Organic Chemistry, Freiestrasse 3, CH-3012 Bern, Switzerland
}

\begin{abstract}
Three graphs that indicate the relative error in peak area when two incompletely resolved peaks are integrated by the vertical drop method are presented. The following cases are investigated: two Gaussian peaks, two exponentially modified peaks, and an exponentially modified peak followed by a Gaussian peak; in each case, the peaks are of equal width. Area ratios range from 1:1 to 10:1, tailing factors of asymmetric peaks range from 1.10 to 2.0, and the resolution of the peak pair varies between 1.5 and 0.75 . Depending on the size ratio, tailing, and resolution, the errors in peak area can be large, for example, up to plus or minus $40 \%$ of the true area within the range of peak pairs investigated. In all cases, the small peak is more impaired than the large one, with impairment depending on the size ratio.
\end{abstract}

\section{Introduction}

In 1972, Snyder (1,2) published two illustrative and useful papers for daily work in the chromatography laboratory. The first publication presented six graphs with large drawings of partially resolved peak pairs. The drawings allowed for the estimation of resolution without the necessity of calculation; in addition, they gave information about true peak height, true peak area, and the purity of collected fractions.

One small figure in Snyder's paper dealt with the area of incompletely resolved peaks that are integrated with the vertical drop method. This is the most widely used integration technique; it involves dividing the peak clusters by dropping a vertical line at the deepest point of the valley down to the baseline. Figure 1 shows this method and its source of error. Both the human eye and the integrator only see the sum curve of the fused peaks; the valley of this curve divides the individual bands at a point that does not represent the accurate peak areas. In Figure 1, as well as in Snyder's drawings, the peaks are of Gaussian (symmetrical) shape. A closer look at Figure 1 shows that the area of the small peak is too small; in the case presented, it is only $93 \%$ of its true area. The area of the large peak is too large, but this error is less than the error for the small peak.
It can be assumed that Snyder's message was not recognized; otherwise, everyone would try to achieve complete resolution of the relevant peaks in a chromatogram. Contemporary integrators and data systems yield nice reports and allow all types of manipulation of the output, therefore calming users. Meanwhile, an important question remains unanswered, although it was discussed briefly by Snyder (1): Given a situation in which peak tailing occurs, is the value of the area determined for the small peak still too small?

This topic has been discussed in the literature from time to time, but again I assume that the results were not presented in a user-friendly form, and they were not detailed enough. Proksch and co-workers (3) published two tables of correction factors for partially resolved Gaussian peaks. Westerberg (4) presented a graph that covered the same problem. Neither paper is easy to understand because the authors did not use the presently established chromatographic terms such as resolution. Grushka and co-workers (5) discussed how overlapping peaks can be discerned with the use of higher central moments (skew and excess), but they found it difficult to realize the theoretically sound results in real chromatograms. Novák and co-workers (6) investigated real chromatographic peaks whose asymmetry was unknown and compared their errors with mathematical models based on Gaussian or triangular peak shapes. Problems associated with quantitation and the detection limit of infrared spectrometric bands were discussed by Vandeginste and De Galan (7); Gaussian-shaped peaks were included in this study. Peichang and co-workers (8) presented peak resolution as a function of peak separation (note the special nomenclature) in the form of graphs, which were not easily understandable. Ogan (9) gave a short discussion of the problem in his chapter on detection in quantitative liquid chromatography. Foley (10) studied a large number of peak pairs of differing size and tailing ratio; he published detailed results about tailed, overlapping peaks of equal area and gave correction factors that allowed the calculation of their true areas. The degree of impurity of fractions from partially overlapped peaks was discussed by Karol (11). Empirical equations for the area determination of unresolved peaks were proposed by $\mathrm{Wu}$ and co-workers (12-14). Finally, inadequate chromato- 
graphic performance and resolution can lead to the "loss" of peaks if a complex mixture is being separated. This problem was studied theoretically and experimentally by Davis (15).

It is the aim of this paper to present graphical illustrations of the relative error that occurs when a pair of poorly resolved peaks is integrated by the vertical drop method. Although it can be assumed that many of the results presented here can already be found in the aforementioned papers, there is still a demand for such graphs because they allow analysts to compare their chromatograms with them and to estimate the error in the area value of a given peak. In this sense, the figures are a completion of the ones published by Snyder (1). An awareness of the relative error associated with area determination is necessary because of the increased use of accreditation schemes (such as ISO 9000) that do not tolerate inaccuracies in quantitative analyses.

\section{Experimental}

This study covers peak pairs of equal width and of differing area ratios, chromatographic resolution, and tailing.

It was necessary to restrict the study to peaks of identical width (a situation often found in programmed analysis, i.e., with temperature programming in gas chromatography and with gradient elution in column liquid chromatography). If ad-

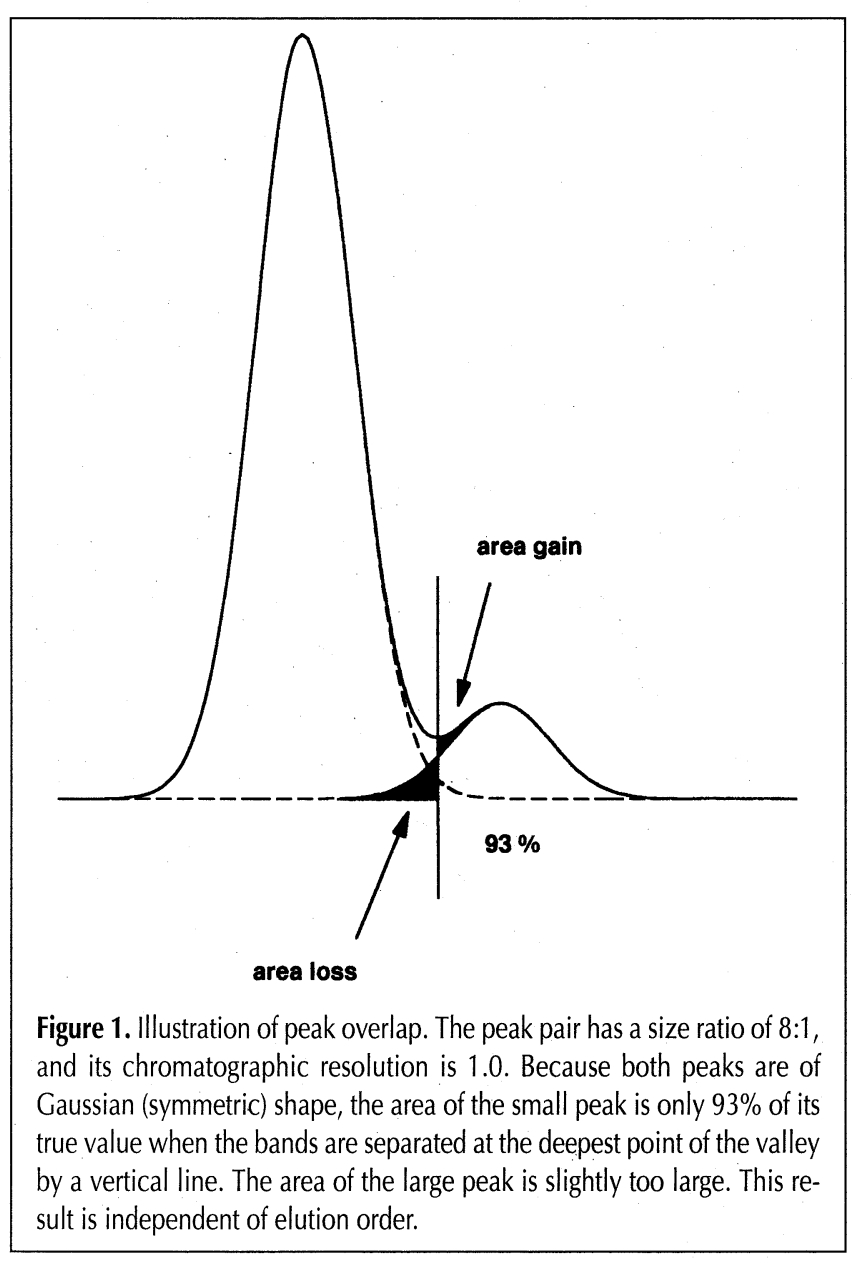

jacent peaks have equal plate numbers (this is the normal case in non-programmed analysis when special effects are absent), their relative width depends on the capacity factors involved (16); especially at low capacity factors, the results deviate from the ones presented here. With increasing capacity factor values, the peak widths equalize, and the results do not deviate any longer.

The area ratios investigated were 1:1, 4:3, 2:1, 4:1, 10:1, and vice versa. For tailed peaks, the results depended on elution order; therefore the position of the smaller peak was important. The chromatographic resolution was set to $1.50,1.25,1.00$, 0.85 , or 0.75 . The asymmetry of tailed peaks was determined as the tailing factor $(T)$, measured at $10 \%$ of peak height, which was the quotient of the trailing "half" and the leading "half" of the peak width. $T$ was varied: $1.10,1.35$, and 1.70 to 2.0 . Because exponentially modified Gaussian-type peaks were used (17), their asymmetry could also be defined as $\tau / \sigma$ (i.e., the time constant of the exponential decay function divided by the standard deviation of the Gaussian function). The $T$ values corresponded to $\tau / \sigma$ values of $0.52,0.98,1.50$, and 1.92 , respectively.

The following cases of partially resolved peaks were investigated: two Gaussian peaks; two exponentially modified peaks, small peak last; two exponentially modified peaks, small peak first; and an exponentially modified peak followed by a Gaussian peak. The opposite of the last case, a Gaussian peak followed by an exponentially modified one, was of much less interest because it is very similar to a Gaussian-Gaussian peak pair.

The peaks were generated by means of Calpeak software (Allied Data Scientific; England), which allowed the creation of peaks of well-defined size (area, width, tailing). This software was designed for the validation of integrators (18), but in addition, it is an excellent research instrument for the investigation of all kinds of chromatograms. The data generated by Calpeak were integrated by a Hewlett-Packard $3390 \mathrm{~A}$ integrator (Hewlett-Packard; Wilmington, DE) by the vertical drop method. This means that the results presented here were not from mathematical investigations but from "pseudo-real" integrations. Because of the way integrators work (it is, for example, necessary to set a threshold), the results may differ slightly but insignificantly from what could be obtained by pure mathematics. The advantage of the technique used for this study is that the results were close to real-world separations but with an accurate knowledge of the true peak areas involved.

All "chromatograms" were integrated twice. The results gave excellent agreement, which is not surprising because they were obtained by the undisturbed interaction of two microprocessors.

\section{Results}

As outlined in the introduction, the results of this study are presented mainly in the form of graphs,' which are selfexplanatory. Figure 2 shows two Gaussian peaks and two exponentially modified peaks, with the small peak last. Figure 3 shows two exponentially modified peaks of equal area, with the small peak first. Figure 4 shows an exponentially modified peak followed by a Gaussian peak. The $x$-axis of all the graphs 



Figure 2. Areas of incompletely resolved peaks: The first column (A) presents the results for two peaks of Gaussian shape (GG), and the other columns $(B, C, D, E)$ present the results for two exponentially modified peaks (EE) of identical tailing, small peak last. The percent of the true area is plotted on the $y$-axis, and the chromatographic resolution is plotted on the $x$-axis. White dots correspond to the first peak, black to the second one. Tailing $(T)$ in- creases from top to bottom, as indicated in the boxes. The true area ratio is also noted in the boxes. All peaks are of identical width. Missing dots indicate that a peak pair could not be resolved by the integrator. The small drawings represent a peak pair of resolution 1.25 with the corresponding asymmetry and size ratio. For Gaussian peaks, the results are independent of elution order. 
indicates the chromatographic resolution (the values are not equidistant), whereas the $y$-axis gives the peak area as a percentage of the true area, as found by the integrator. Each graph contains a small drawing of the relevant peak pair and how it presents itself at a resolution of 1.25. The first peak is white, with white dots representing its area, whereas the second peak is black with black dots. The type of peak pair, its size ratio, and the tailing $(T)$ of asymmetric peaks is indicated in the legend. Empty space in the graphs marks peak pairs that could not be resolved by the integrator.

The numeric values that are the basis of the graphs have been tabulated and are available from the author on request.

When both peaks differ in their true area, then the graph is asymmetric. The degree of asymmetry directly reflects the area ratio: If this ratio is 4:1, then the relative error of the large peak is $1 / 4$ of the error of the small peak because the same absolute amount of area has been removed from or added to the peaks.

\section{Two Gaussian peaks}

In Figure 2A, the area of the small peak is too small, as noted by Snyder (1). The reason for this is obvious from Figure 1. If deviations from this behavior are found, the effects are small and are a result of the aforementioned features of integrators. Because of the symmetry of the peaks, the results are equally valid if the small peak is eluted first. A comparison with the other figures makes it clear that the errors are markedly lower than in any case where tailed peaks are involved. Two Gaussian peaks of identical area and width are not included in Figure 2. This is the only case that, in principle, can be integrated correctly. With the simulation used in this work, it was found that the integrator noted an area ratio of 99.9:100.1 (first:second peak).

\section{Two exponentially modified peaks, small peak last}

The area of the small peak in Figures 2B-2E is too large because it benefits from the "tail" of its predecessor. In some cases with tailing of 1.0, the area of the small peak is too small; this effect concerns peak pairs that are at the limit of recognition for the integrator (i.e., there are not two distinct peaks; the second one is present merely as a shoulder).

\section{Two exponentially modified peaks of equal size}

Because of the reason already mentioned, the second peak has a larger area than the first one in Figure 3A. If the tailing is pronounced, this can lead to the optical illusion that the later eluted band is really larger than the earlier one. The integrator will confirm this conviction (Figure 5).

\section{Two exponentially modified peaks, small peak first}

Because of the same reason (but now with the opposite effect), the small peak is too small (Figures 3B-3E). The detectability of the minor band is better than if it is eluted last (there is less empty space than in Figures 2B-2E), but its error can reach huge values that exceed $40 \%$.

\section{Exponentially modified peak followed by a smaller Gaussian peak}

If a tailed and a Gaussian peak overlap, interesting effects can be expected if the tailed peak is eluted first. (Otherwise the results are similar to the Gaussian-Gaussian case.) If the exponentially modified peak is smaller than the Gaussian one, its area will be too small, and the area of the Gaussian peak will be slightly too large; the results are similar to those shown in Figure 3. In Figure 4, the case of a tailed peak followed by a smaller symmetrical one is studied in detail. The area of the second peak is too large, but the error is less than in the case of two tailed peaks (Figure 2). This could be explained by the "better" integration behavior of a Gaussian peak, the end of which is better defined than if it were tailed. Deviations from the general trend are due to recognition problems for the integrator.

\section{Discussion}

The results show that the determination of the area of incompletely resolved peaks can be highly problematic, especially if the area ratios of the bands involved are large and if the peaks are tailed. The best remedies against these errors are the improvement of resolution and the suppression of tailing. Although it can be difficult to comply with these requisitions, analysts should concentrate their efforts on this task. The most promising means to improve resolution are the enlargement of chromatographic selectivity and theoretical plate number. Tailing can be reduced by diminishing dead volumes, looking for a more adequate separation system (to reduce chemical tailing), and using programmed elution (temperature program or solvent gradient).

If it is impossible to improve resolution or decrease tailing, it is a good practice to mimic the peak size ratios as close as possible with the calibration chromatogram. Because the relative error depends on size ratio, it is not good advice to calibrate with a single standing peak or with a pair of incompletely resolved peaks that deviate markedly in size ratio from what is found in the sample chromatogram.

One may question whether peak height determination would be better than peak area determination. In regard to this point, Foley (10) noted that for tailed peaks of equal area, the errors in peak height are less than those in peak area. In a study (19) that included peak pairs with a 10:1 area ratio, it was found that the peak height measurement was more accurate than area measurement when the small peak was eluted first, irrespective of the tailing involved. When the small peak was eluted last, a height determination was only superior if tailing was absent. When the peaks were tailed, the error in the quantitation of a small peak, which was eluted second, was large with both techniques.

The best solution to the problem of incompletely resolved peaks is mathematical fitting. Appropriate software packages for personal computers are commercially available: PeakFit from Jandel Scientific (San Rafael, CA) and PeakCalc (20) from Reh EDV Consulting (Spabrücken, Germany). Because these programs are new, data about their usefulness in everyday work in a chromatography laboratory is not yet available.

Chemometrics can help to resolve two overlapping peaks if 








Figure 4. Areas of incompletely resolved peaks: Exponentially modified peaks followed by Gaussian peaks are presented. The percent of the true area is plotted on the $y$-axis, and the chromatographic resolution is plotted on the $x$-axis. White dots correspond to the first peak, black to the second one. Tailing $(T)$ increases from top to bottom as indicated in the boxes. The true area ratio is also noted in the boxes. Missing dots indicate that a peak pair could not be resolved by the integrator. The small drawings represent a peak pair of resolution 1.25 with the corresponding asymmetry and size ratio. 
the detector properties of the compounds differ. In highperformance liquid chromatography (HPLC), the use of a diode-array detector is common, and this allows improved quantitation (21). In gas chromatography, the coupling of two different detectors such as photo-ionization and flame-ionization detectors, allows quantitation (22). These techniques fail, of course, if the compounds have identical properties (e.g., enantiomers).

Another problem is the incomplete resolution of more than two peaks. This can be reduced to the case of three fused peaks, but even then, it is an endless struggle because innumerable combinations of large and small, symmetrical and tailed peaks can be involved. However, the results presented in this paper can be an aid because it is possible to estimate the error of a middle peak by adding the errors found from the influence of the preceding peak plus the influence of the following peak.

A possible extension of this study would be the investigation of peak pairs of markedly unequal peak width, for example, by selective peak compression due to special effects during the chromatographic separation.

\section{Chromatographic example}

As an illustration, Figure 6 represents the incomplete HPLC separation of naphthalene and anthracene at two different detection wavelengths. At $295 \mathrm{~nm}$, naphthalene has markedly higher molar absorptivity than anthracene, and the peak area ratio is approximately $2: 1$ when identical amounts are present in the mixture. At $310 \mathrm{~nm}$, the ultraviolet absorption properties are different, giving an area ratio of approximately 1:4. The peaks were asymmetric, with tailing of 1.5 , and their resolution was 0.8 when eluted with a strong mobile phase (water-methanol, 1:3). Their areas were compared with the values obtained at good resolution (water-methanol, 4:6), and they confirmed the results obtained by artificial peaks.

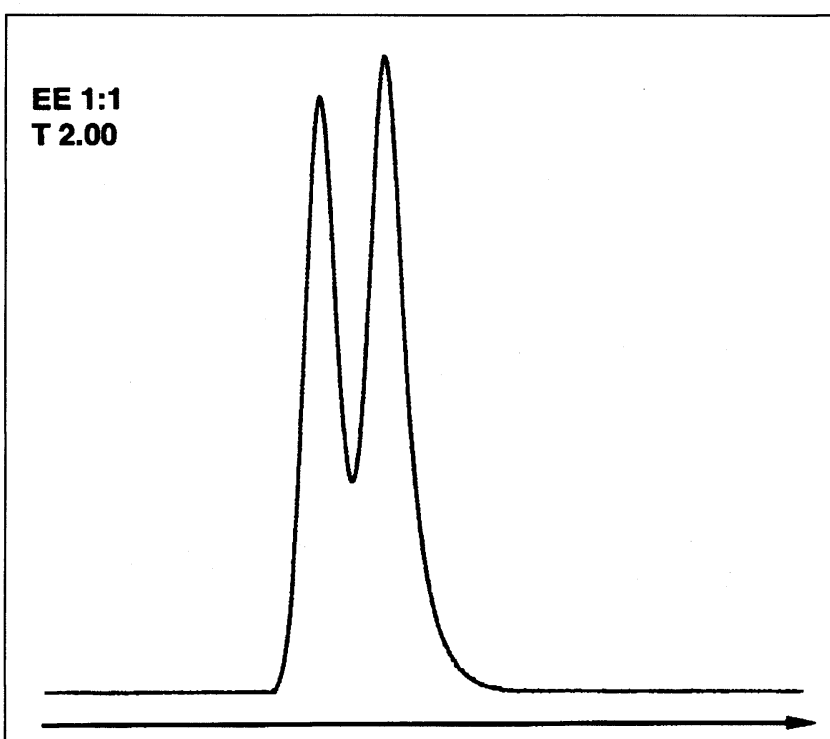

Figure 5. Two asymmetric peaks of equal area, both with tailing of 2.0. The chromatographic resolution is 1.0. The tailing leads to the impression that the second peak is larger in area.

\section{Conclusion}

It was found that integration errors obtained from the incomplete resolution of chromatographic peaks can be large. Errors increase with increasing size ratio of two fused peaks, increasing tailing, and decreasing resolution. Within the range of parameters investigated here (size ratio up to 10:1, tailing up to 2.0 , resolution down to 0.75 ), the relative error can reach a $40 \%$ deviation in peak area, as shown in Figures 2, 3, and 4.

\section{Acknowledgment}

I am indebted to Dr. Joe Foley of Villanova University in Pennsylvania for stimulating discussions. The Calpeak software was a gift from Dr. Fritz Erni, Sandoz AG, in Basel, Switzerland.

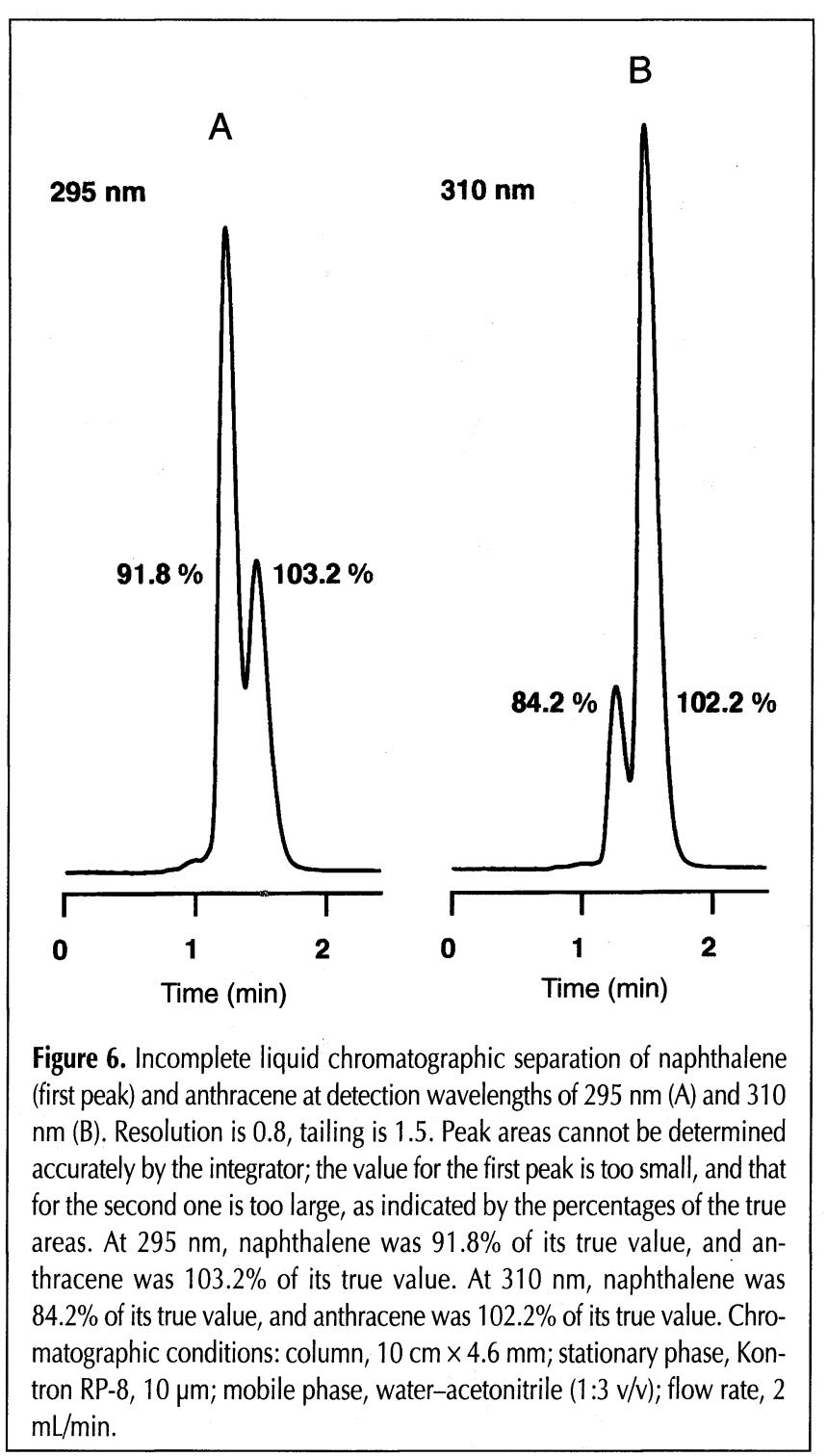




\section{References}

1. L.R. Snyder. A rapid approach to selecting the best experimental conditions for high-speed liquid column chromatography. Part I: Estimating initial sample resolution and the final resolution required by a given problem. J. Chromatogr. Sci. 10: 200-12 (1972).

2. L.R. Snyder. A rapid approach to selecting the best experimental conditions for high-speed liquid column chromatography. Part II: Estimating column length, operating pressure and separation time for some required sample resolution. J. Chromatogr. Sci. 10: 369-79 (1972).

3. E. Proksch, H. Bruneder, and V. Granzner. Correction factors for the quantitative evaluation of overlapping gas chromatographic peaks. J. Chromatogr. Sci. 7: 473-83 (1969).

4. A.W. Westerberg. Detection and resolution of overlapped peaks for an on-line computer system for gas chromatographs. Anal. Chem. 41: 1770-77 (1969).

5. E. Grushka, M.N. Myers, and J.C. Giddings. Moments analysis for the discernment of overlapping chromatographic peaks. Anal. Chem. 42: 21-26 (1970).

6. J. Novák, K. Petrovic, and S. Wicar. Efficacy of the corrections applied in the resolution of overlapping chromatographic peaks by the perpendicular drop method. J. Chromatogr. 55: 221-29 (1971).

7. B.G.M. Vandeginste and L. De Galan. Critical evaluation of curve fitting in infrared spectrometry. Anal: Chem. 47: 2124-32 (1975).

8. L. Peichang, L. Xiuzhen, and Z. Yukuei. The theoretical basis of column chromatography in multicomponent separations. Part 1: Analytical requirements and peak resolution. Development of a high performance column system. J. High Res. Chromatogr. 3: 551-67 (1980).

9. K. Ogan. Detection in quantitative liquid chromatography. In Quantitative Analysis Using Chromatographic Techniques. E. Katz, Ed. John Wiley \& Sons, Chichester, UK, 1987, pp 31-61.
10. J.P. Foley. Systematic errors in the measurement of peak area and peak height for overlapping peaks. J. Chromatogr. 384: 301-13 (1987).

11. P.J. Karol. Column resolution and peak overlap. J. Chromatogr. Sci. 27: 578-82 (1989).

12. N.S. Wu and G.H. Gu. Peak area measurement of severely overlapped peaks. Chromatographia 32: 373-78 (1991).

13. N.S. Wu and W. Wei. Empirical equation for relative non-overlap. Chromatographia 34: 450-52 (1992).

14. W. Wei, N.S. Wu, and X.H. Jiang. New approach for area determination of an overlapped pair of chromatographic peaks. J. Chromatogr. 623: 366-70 (1992).

15. J.M. Davis. Statistical considerations in chromatographic separations. J. High Res. Chromatogr. 14: 501-502 (1991).

16. V.R. Meyer. Use of chromatographic peak-height ratios for quantitative analysis: Application to the separation of enantiomers. J. Chromatogr. 623: 371-74 (1992).

17. J.P. Foley and J.G. Dorsey. A review of the exponentially modified Gaussian (EMG) function: Evaluation and subsequent calculation of universal data. J. Chromatogr. Sci. 22: 40-46 (1984).

18. N. Dyson. The validation of integrators and computers for chromatographic measurements. Int. Lab. 22(6): 38-46 (1992).

19. V.R. Meyer. Chromatographic integration errors: A closer look at a small peak. LC-GC Int. 7: 94-100 (1994).

20. E. Reh. An algorithm for peak-shape analysis for differentiating unresolved peaks in chromatography. Trends Anal. Chem. 12: 192-94 (1993).

21. J.K. Strasters. Chemometrics and photodiode array detection. In Diode Array Detection in HPLC. L. Huber and S.A. George, Eds. Marcel Dekker, New York, NY, 1993, pp 127-61.

22. F.L. Herman. Tandem detectors to quantitate overlapping chromatographic peaks. Anal. Chem. 65: 1023-27 (1993).

Manuscript received October 6, 1993; revision received April 4, 1994. 